\title{
Articular Chondrocytes from Animals with a Dermatan Sulfate Storage Disease Undergo a High Rate of Apoptosis and Release Nitric Oxide and Inflammatory Cytokines: A Possible Mechanism Underlying Degenerative Joint Disease in the Mucopolysaccharidoses
}

\author{
Calogera M. Simonaro, Mark E. Haskins, and Edward H. Schuchman \\ Department of Human Genetics (CMS, EHS) and Institute for Gene Therapy and Molecular Medicine (EHS), Mount \\ Sinai School of Medicine, New York, New York; and Department of Pathobiology (MEH), University of Pennsylvania \\ School of Veterinary Medicine, Philadelphia, Pennsylvania
}

\begin{abstract}
SUMMARY: Mucopolysaccharidosis (MPS) Type VI (Maroteaux-Lamy Disease) is the lysosomal storage disease characterized by deficient arylsulfatase B activity and the resultant accumulation of dermatan sulfate-containing glycosaminoglycans (GAGs). A major feature of this and other MPS disorders is abnormal cartilage and bone development leading to short stature, dysostosis multiplex, and degenerative joint disease. To investigate the underlying cause(s) of degenerative joint disease in the MPS disorders, articular cartilage and cultured articular chondrocytes were examined from rats and cats with MPS VI. An age-progressive increase in the number of apoptotic chondrocytes was identified in the MPS animals by terminal transferase nick-end translation (TUNEL) staining and by immunohistochemical staining with anti-poly (ADP-ribose) polymerase (PARP) antibodies. Articular chondrocytes grown from these animals also released more nitric oxide (NO) and tumor necrosis factor alpha $(\mathrm{TNF}-\alpha)$ into the culture media than did control chondrocytes. Notably, dermatan sulfate, the GAG that accumulates in MPS VI cells, induced NO release from normal chondrocytes, suggesting that GAG accumulation was responsible, in part, for the enhanced cell death in the MPS cells. Coculture of normal chondrocytes with MPS VI cells reduced the amount of NO release, presumably because of the release of arylsulfatase B by the normal cells and reuptake by the mutant cells. As a result of the enhanced chondrocyte death, marked proteoglycan and collagen depletion was observed in the MPS articular cartilage matrix. These results demonstrate that MPS VI articular chondrocytes undergo cell death at a higher rate than normal cells, because of either increased levels of dermatan sulfate and/or the presence of inflammatory cytokines in the MPS joints. In turn, this leads to abnormal cartilage matrix homeostasis in the MPS individuals, which further exacerbates the joint deformities characteristic of these disorders. (Lab Invest 2001, 81:1319-1328).
\end{abstract}

$T$ he mucopolysaccharidoses (MPS) are a family of inherited lysosomal storage disorders resulting from the deficient activities of specific lysosomal enzymes required for the step-wise degradation of glycosaminoglycans (GAGs) (Neufeld and Muenzer, 2001). To date, 11 distinct MPS enzyme deficiency disorders have been described. In each disease the primary enzyme deficiency leads to the accumulation of partially degraded GAGs within the lysosomes of the patient's cells, resulting in an array of clinical features that progress with age. For example, in MPS type VI (Maroteaux-Lamy disease), a deficiency of

Received June 25, 2001.

This work was supported by Grant RO1 DK 25759 from the National Institutes of Health.

Address reprint requests to: Dr. Calogera M. Simonaro, Department of Human Genetics, Mount Sinai School of Medicine, 1425 Madison Avenue, New York, New York 10029. E-mail: Calogera.Simonaro@mssm.edu arylsulfatase B (ASB; N-acetyl galactosamine 4-sulfatase) activity results in the accumulation of dermatan sulfate. MPS type VI is characterized by short stature, dysostosis multiplex, cranial and tracheal abnormalities, cornea and cardiovascular defects, and degenerative joint disease (Neufeld and Muenzer, 2001).

Histologically, chondrocytes from individuals with the MPS disorders are filled with enlarged, GAGcontaining vacuoles that obscure other organelles and displace and deform the nucleus. Clusters of distended and rounded chondrocytes are thereby formed, disrupting the normal columnar architecture of the growth plate cartilage and presumably leading, in part, to the bone growth abnormalities (Abreu et al, 1995; Norrdin et al, 1993). Osteoblast and osteocyte functions seem to be preserved in MPS individuals, and bone formation and demineralization occurs at a normal rate (Abreu et al, 1995). Abnormalities of the 
articular chondrocytes and cartilage are also likely to play an important role in the degenerative joint disease associated with the MPS disorders, but as with the pathogenesis of the bone growth abnormalities, the nature of the degenerative joint disease in the MPS disorders is poorly understood. The articular cartilage is initially thicker in MPS VI animals (as is the synovium), suggesting that there may be abnormalities in articular cartilage matrix formation and function (Crawley et al, 1997).

Apoptosis is a normal and well-characterized process in growth plate chondrocytes. During the formation of long bones, chondrocytes in the epiphyseal growth plates proliferate in columns toward the diaphysis of the bone, becoming hypertrophied as they deposit cartilage matrix and eventually undergoing apoptosis (Gerber et al, 1999; Hashimoto et al, 1999). In contrast, apoptosis of articular chondrocytes has not been described in healthy individuals, although abnormal formation of apoptotic bodies has been associated with pathogenic events, such as osteoarthritis (OA) (Hashimoto et al 1998), and/or the mechanical loading of normal joints.

In the present study, we examined apoptosis in the articular cartilage and cultured articular chondrocytes of MPS VI rats and cats. We demonstrate agedependent morphological and biochemical changes associated with increased apoptosis in the articular cartilage of the affected animals, but not age-matched healthy animals. We also observed a depletion of proteoglycans and total collagen in the MPS VI cartilage matrix, suggesting that a direct consequence of the enhanced apoptosis is abnormal cartilage matrix composition. A hypothesis explaining the joint abnormalities in the MPS disorders is proposed whereby articular chondrocyte cell death is induced by GAG accumulation within these cells and/or the release of inflammatory cytokines caused by mechanical stress imposed on the MPS joints. The diminished function and increase in cell death of the MPS VI chondrocytes leads to abnormal articular cartilage matrix composition, ultimately causing degeneration of the articular surfaces and further joint defects.

\section{Results}

\section{Assessment of Apoptosis in Primary MPS VI Rat Chondrocytes and Articular Cartilage}

Primary chondrocyte cultures were established from the articular cartilage (proximal tibia) of approximately 6-month-old MPS VI and healthy rats ( $n=4$ each) and subjected to terminal transferase nick-end translation (TUNEL) staining. After 5 days in culture, $7.5 \%$ of the normal cells were TUNEL-positive, whereas $30.5 \%$ were positive in the MPS VI cultures (not shown).

To further investigate these in vitro findings, articular cartilage from MPS VI rats at various ages (2 weeks to 12 months) was assessed in situ by TUNEL staining using both colorimetric and fluorometric detection systems. As illustrated in Figure 1, many of the articular chondrocytes in the proximal tibia of 6-month-old MPS VI rats were found to be TUNEL-positive when compared with agematched healthy rats (compare Panels A and B). Similar findings were observed in the proximal tibia of 1-year-old MPS VI cats (not shown). Increased apoptosis in the tibia was first observed at 3 months in the MPS VI rats and at 9 to 12 months in MPS VI cats (Fig. 2, A and B, respectively), and progressed with age. To assess whether enhanced chondrocyte apoptosis occurred in other synovial joints, the proximal humeri from 6-monthold MPS VI rats also were examined. As shown in Figure 1, Panels $C$ and $D$, the articular chondrocytes from the proximal head of the MPS VI humeri also had markedly enhanced apoptosis compared with age-matched controls.

TUNEL staining detects late-stage DNA fragmentation and is one measure of apoptosis. To confirm the apoptosis indexes obtained with TUNEL assays, an immunocytochemical study was performed using antipoly (ADP-ribose) polymerase (PARP, 85-kd fragment) polyclonal antibodies. The $85-\mathrm{kd}$ PARP is a product of caspase-3 activity and a commonly used marker for apoptosis. As can be seen in Figure 3, the MPS VI rat tibia and humeri also had enhanced immunostaining with anti-PARP antibodies compared with agematched controls, confirming the TUNEL results.

\section{TNF- $\alpha$ and NO Production by Normal and MPS VI Chondrocytes}

To investigate whether the enhanced apoptosis observed in the MPS VI cartilage was associated with cytokine-induced nitric oxide (NO) production, primary chondrocyte cultures were established from the proximal tibia of MPS VI rats, and the levels of TNF- $\alpha$ and nitrite were determined in the culture media. As shown in Figure 4, both compounds were markedly increased in the media of MPS VI cells compared with normal cells, and the levels were significantly greater in older MPS animals (8 months) compared with young (3 months). Similar nitrite results were obtained in cells grown from the proximal tibia of MPS VI cats ranging in age from 6 months to 5 years (Fig. 5).

\section{Effect of LPS and Dermatan Sulfate on NO Production in Normal and MPS VI Chondrocytes}

Lipopolysaccharide (LPS) induces NO production in many cell types, including chondrocytes (Palmer et al, 1993; Stadler et al, 1991). Dermatan sulfate, the GAG that accumulates in MPS VI cells, is a complex polysaccharide with structural homologies to LPS. Therefore, we compared the effects of LPS and dermatan sulfate on NO release from normal and MPS VI articular chondrocytes (Fig. 6). Notably, dermatan sulfate stimulated NO release from normal cells to an even greater degree than LPS. Furthermore, in MPS VI cells, the baseline level of nitrite in the media was greater than normal, confirming the results shown above in Figure 4B, and both LPS and dermatan sulfate enhanced this effect. 

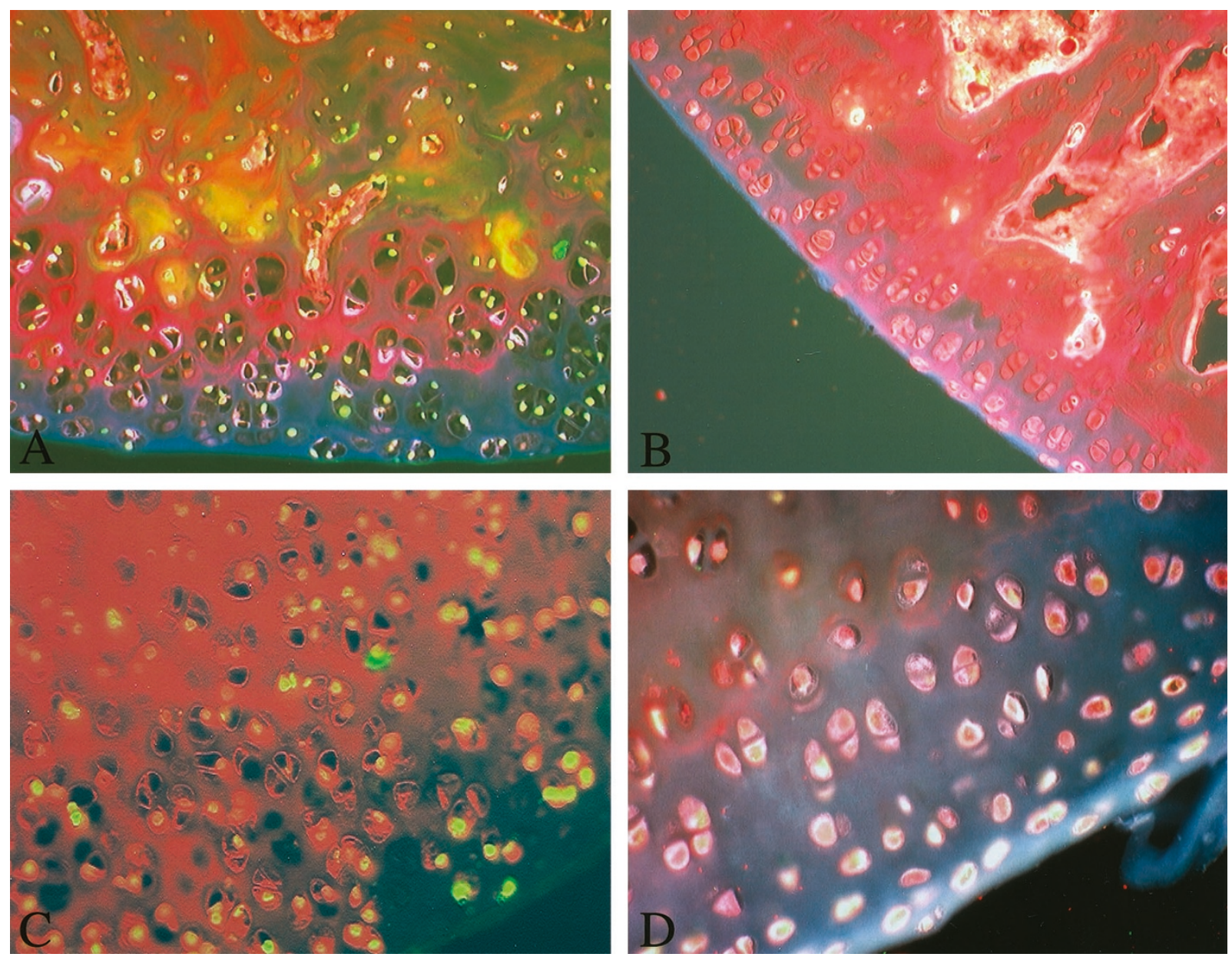

Figure 1.

Fluorescence-based terminal transferase nick-end translation (TUNEL) staining of articular cartilage from mucopolysaccharidosis type VI (MPS VI) rats. Articular cartilage and epiphyseal bone from the tibia of MPS VI and normal rats are shown in Panels A and B, respectively (magnification, $\times 20$ ). Note the presence of fluorescent nuclei in the MPS VI animals (A). C and D, TUNEL staining was repeated on the articular cartilage from the proximal end of the humeri (magnification, $\times 40$ ). Once again, note the fluorescent nuclei in the MPS VI rats (C) compared with normal animals (D).

\section{Effect of Coculturing Normal and MPS VI Chondrocytes on NO Release}

To determine whether arylsulfatase $B$ that was secreted by normal chondrocytes could reduce NO release by MPS VI cells, a coculture experiment was carried out. As shown in Figure 7, initially the NO levels in the media of co-cultured normal and MPS VI cells (gray bars) were approximately the sum of those found in the normal (white bars) and MPS VI (black bars) control cultures. However, by 80 hours these levels had been reduced to almost normal. Also note that during this same time period the NO levels in the control cultures did not vary significantly. After 80 hours the cells were harvested from the wells and arylsulfatase B (ASB) activity was determined. The levels of ASB in the cocultured MPS VI cells were approximately $60 \%$ of normal, indicating significant release of the enzyme by normal cells and reuptake by the MPS VI cells (not shown).

\section{Analysis of MPS VI Articular Cartilage Matrix Abnormalities}

Chondrocyte apoptosis has been associated with proteoglycan depletion in osteoarthritis $(\mathrm{OA})$, leading to cartilage degradation. Safranin-O and alcian blue staining, both of which have been used to detect proteoglycan depletion in OA (Hashimoto et al, 1998), were used to assess proteoglycans in the articular cartilage and underlying bone of MPS VI animals. As illustrated in Figure 8, areas of the MPS VI rat cartilage and bone stained much less intensely with safranin-O than in healthy animals, indicating that matrix proteoglycan depletion is a feature of this MPS disorder. Similar observations were obtained using alcian blue in both the MPS VI rats and cats (not shown).

Collagen is a major component of articular cartilage and is synthesized and degraded by chondrocytes (Mayne, 1989). In the MPS disorders, where enhanced chondrocyte apoptosis was observed, we hypothesized that collagen metabolism might also be affected because of decreased collagen synthesis or aberrant release of collagen-processing proteases into the extracellular matrix. Again, similar abnormalities in collagen processing have been made in OA (Billinghurst et al, 1997). Articular cartilage was obtained from the proximal tibia of healthy and MPS VI rats, and the total collagen content was quantified by measuring hydroxyproline. As 

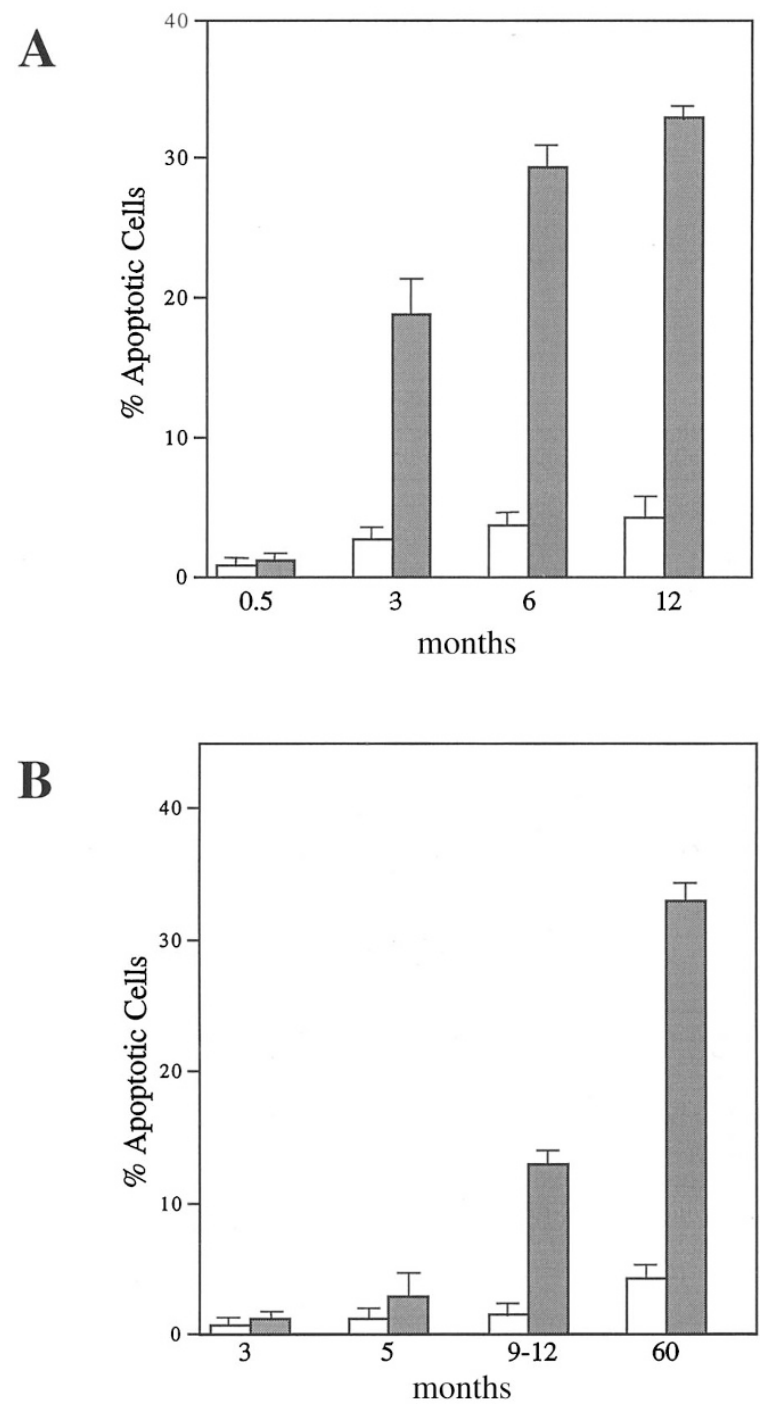

Figure 2.

Age-dependent accumulation of TUNEL-positive cells in the articular cartilage of MPS VI rats and cats. Saggital sections were prepared from rat and cat proximal tibia, and the chondrocytes were analyzed by TUNEL staining. Two hundred cells were counted for each section. In each age group, multiple sections (2-4) for at least two animals were scored. The height of the columns indicates the mean number of apoptotic cells identified. The error bars show the standard error of the mean. Gray columns represent MPS VI animals; open columns indicate healthy animals. Panels A and B depict data for the MPS VI rats and cats, respectively. Percent apoptosis represents the percent of TUNEL-positive cells.

shown in Figure 9, significant $(p<0.05)$ collagen depletion was observed in the MPS VI cartilage compared with cartilage from healthy animals.

\section{Discussion}

The present study was designed to gain a better understanding of the mechanism(s) underlying degenerative joint disease in the MPS disorders. Four general observations were made. First, and perhaps most importantly, an age-dependent increase in articular chondrocyte apoptosis, as documented by positive TUNEL staining and the presence of PARP-positive cells, was observed in several synovial joints of both MPS VI rats and cats.
Consistent with this observation, cultured MPS VI articular chondrocytes released more NO than normal cells. Second, dermatan sulfate, the GAG that accumulates in MPS VI cells, induced NO production in normal cells to a degree similar to or greater than LPS and enhanced the release of NO from MPS VI cells. Third, the production of the inflammatory cytokine, $\mathrm{TNF}-\alpha$, was markedly elevated in MPS VI chondrocytes compared with normal cells. And fourth, as a result of these chondrocyte abnormalities, defects in the articular cartilage matrix composition were observed in the MPS VI animals, including a generalized proteoglycan and collagen depletion.

Although apoptotic chondrocytes have been described in normal growth plate cartilage during endochondral ossification and chondrogenesis, they are not a common feature of articular chondrocytes. Indeed, in only one other condition has a similar finding been reported, ie, OA. Enhanced chondrocyte apoptosis has been observed in articular cartilage from patients with $\mathrm{OA}$ and plays an important role in the cartilage destruction and degenerative joint disease characteristic of this condition (Lotz et al, 1999). The pathways leading to chondrocyte apoptosis in OA remain unknown, although elevated $\mathrm{NO}$ has been associated with apoptosis of normal chondrocytes following stimulation with IL-1 or LPS and OA cartilage expresses a higher level of inducible NO synthetase than does normal cartilage (Lotz et al, 1999).

The fact that articular chondrocytes from the MPS VI animals had significantly higher rates of apoptosis than those of age-matched controls suggests that the accumulating GAG fragments might be directly inducing apoptosis. Notably, dermatan sulfate has many structural similarities to bacterial LPS, a potent and well-characterized apoptosis inducer (Firestein, 1999). Indeed, our data showed that dermatan sulfate could elicit a similar or greater apoptosis effect (as measured by NO production) on chondrocytes than LPS, consistent with the hypothesis that the accumulating GAGs in the MPS disorders endow these cells with an enhanced propensity to cell death. It has also been suggested that lysosomal hydrolases-eg, various proteases (Sakahira et al, 1998), sphingomyelinase (Santana et al, 1996), and ceramidase (Spiegel et al, 1996)_may play roles in the complex signaling events leading to apoptosis. Thus, generalized lysosomal dysfunction resulting from the primary GAG accumulation may also be inducing articular chondrocyte cell death. Consistent with these observations, coculture of normal chondrocytes with MPS VI cells reduced the amount of NO release by the mutant cells, presumably because of ASB release from the normal cells and reuptake by the MPS VI cells.

In our experience the enhanced apoptosis in the MPS animals was specific for articular chondrocytes and was not found in chondrocytes from the hyaline cartilage of the trachea or other connective tissue cells, which also accumulate dermatan sulfate (CM Simonaro, unpublished data). This suggests that apoptosis is dependent on close interactions with the surrounding articular cartilage environment and is not 

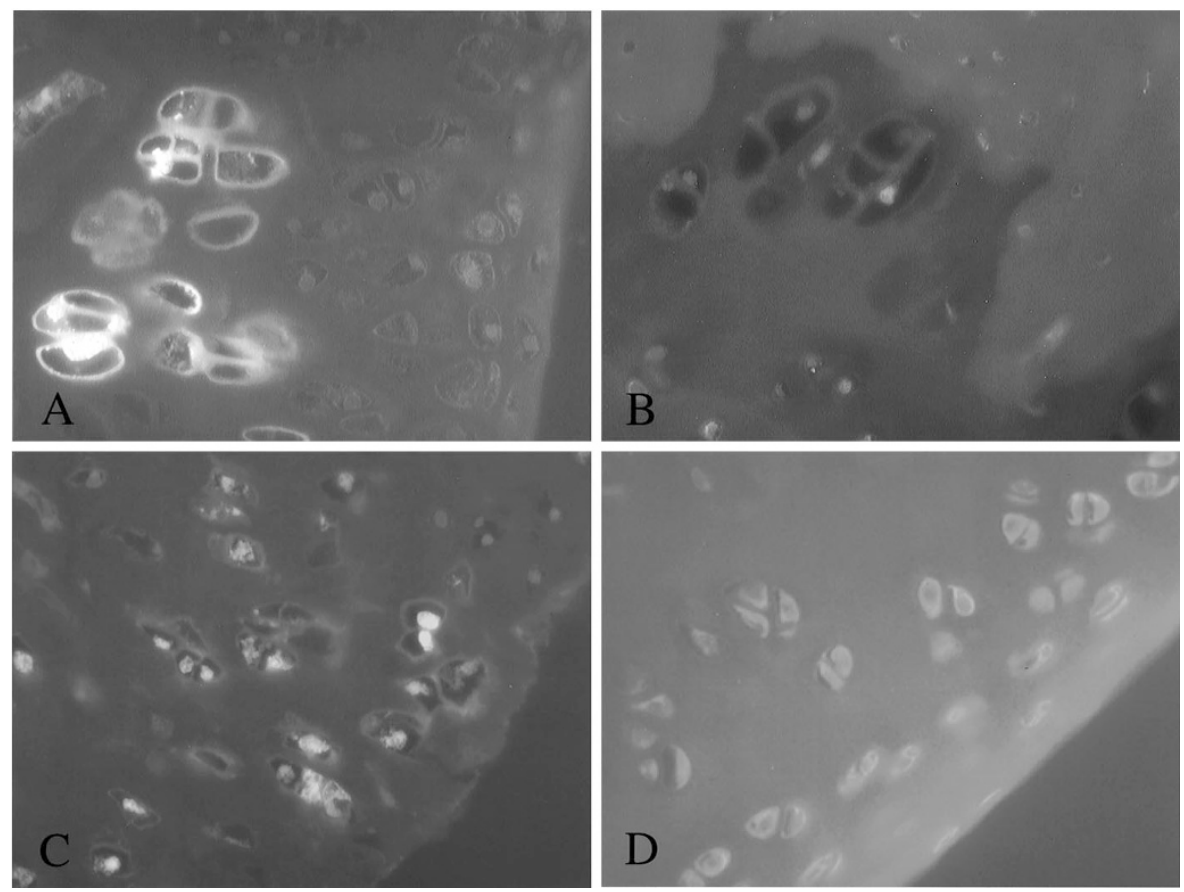

Figure 3.

Poly (ADP-ribose) polymerase (PARP) immunostaining of the articular cartilage of MPS VI rats. Rabbit anti-human PARP polyclonal antibodies were used to visualize the early events of apoptosis in healthy and MPS VI rats. Sagittal sections of the proximal ends of the tibia and humeri from 6-month-old rats were used. A and B, MPS VI and normal rat tibia, respectively; $C$ and D, MPS VI and normal rat humeri, respectively. Note the strongly fluorescent chondrocytes in both MPS VI articular surfaces. Magnification, $\times 60$.

due to GAG accumulation alone. One unique feature of articular cartilage in the MPS individuals is its possible exposure to abnormal mechanical forces that may arise from the joint deformities in these patients. Such mechanical forces can lead to the release of inflammatory cytokines that exacerbate cell death, as in OA (Hamerman et al, 1999). We found that MPS VI articular chondrocytes released markedly elevated levels of TNF- $\alpha$ compared with normal cells, consistent with the hypothesis that inflammation is also involved in the pathogenesis of joint disease in this MPS disorder.

Because of the increased numbers of dead or dying chondrocytes in the articular cartilage of MPS VI animals, we predicted that there might also be a resulting abnormal matrix composition in these individuals. Indeed, we found a depletion of total proteoglycans and collagen in the MPS articular matrix compared with normal. In OA, the only other condition where enhanced articular chondrocyte apoptosis has been found, abnormal collagen metabolism has also been observed (Lotz, 1999). Collagen is the major protein component of the cartilage matrix and is required for tensile strength and matrix organization. Notably, a relationship between collagen and apoptosis has also previously been implicated through the use of gene-targeting technology. Mice in which the type II collagen gene was inactivated by gene targeting had enhanced chondrocyte apoptosis (Yang et al, 1997), again suggesting that cell-matrix interactions play a key role in the normal program of chondrocyte cell death.
Another phenomenon obvious in the MPS VI animals was depletion of the proteoglycans in the cartilage matrix and underlying bone, as visualized by safranin-O and alcian blue staining. The OA literature may also provide analogies to the MPS conditions in this regard because OA cartilage with apoptotic chondrocytes also has reduced proteoglycan content. This observation is not surprising because chondrocytes are the major source of new cartilage matrix components and any condition that leads to a depletion in the cellularity of cartilage and/or to reduced function of chondrocytes is likely to result in such a phenomenon.

From these studies a theory may be proposed to explain the pathogenesis of degenerative joint disease in MPS VI (Fig. 10). We propose that the ageprogressive accumulation of dermatan sulfate fragments provides an enhanced propensity for cell death in the articular chondrocytes of MPS VI individuals. This is compounded by the mechanical forces placed on joints, which lead to inflammatory responses. Together, the progressive GAG accumulation and inflammation lead to articular cartilage matrix abnormalities, causing further destruction of the joints. The varying degree of skeletal involvement in the various MPS disorders may depend, in part, on the type of GAG fragments that are accumulating and being released into the surrounding matrix upon chondrocyte cell death, as well as on the degree of mechanical stress placed on the joints. A study comparing the effects of individual GAGs on chondrocyte cell death is currently underway. 
A

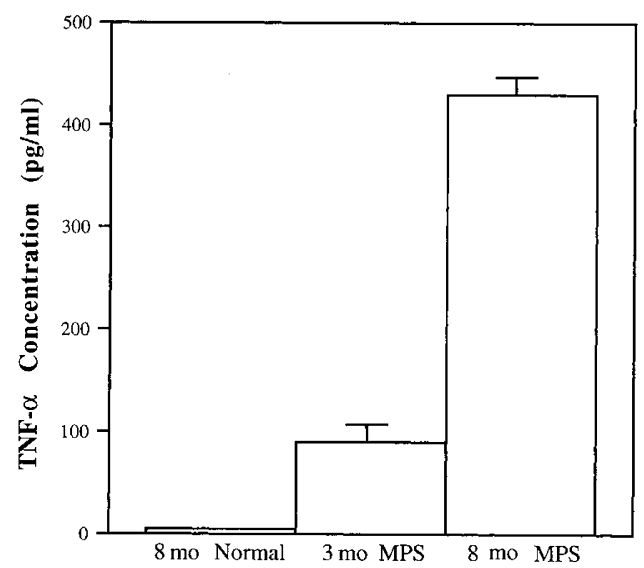

B

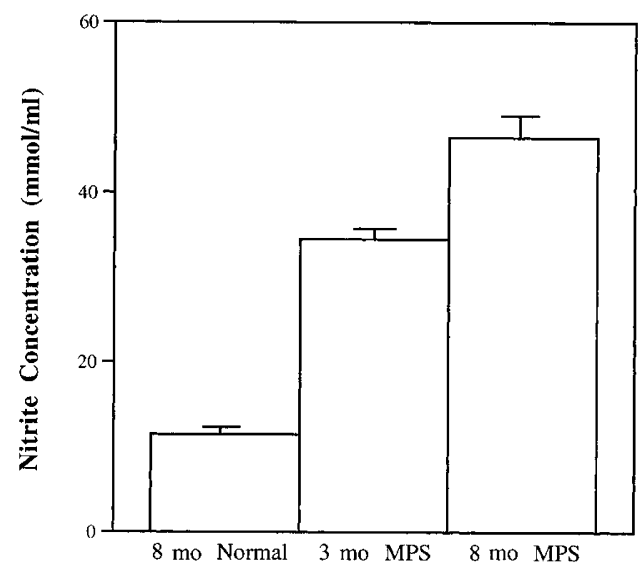

Figure 4.

Tumor necrosis factor alpha (TNF- $\alpha$ ) and nitric oxide (NO) formation in the media of MPS VI rat articular chondrocytes. Articular chondrocytes were obtained and grown from age-matched normal and MPS VI rats as described in "Materials and Methods," and the nitrite and TNF- $\alpha$ concentrations were determined in the culture media after 24 hours. For each age group, chondrocyte cultures from at least three different animals were analyzed in duplicate. The height of the columns represents the mean values obtained, and error bars show the standard error of the mean.

Finally, these findings suggest new approaches for the management and treatment of MPS VI and perhaps other MPS disorders. To date, efforts to treat the MPS conditions have relied on the delivery of the relevant normal enzyme to various sites of pathology in vivo by enzyme replacement (Bielicki et al, 1999; Kakkis et al, 1996; Turner et al, 1999), cell transplantation (Peters et al, 1998; Simonaro et al, 1997), or gene transfer approaches (Daly et al, 1999; Ohashi et al, 1999; Simonaro et al, 1999). Although these strategies have been effective to varying degrees, their effects on skeletal and joint pathology have generally been limited. In part, this may be due to the unique and difficult challenge of delivering recombinant enzymes or enzyme-expressing cells to the bone growth plates and articular cartilage. The current studies suggest that an alternative approach to treatment may be to prevent or reduce the articular chondrocyte apoptosis in these disorders by some means other than enzyme delivery. Small-molecule inhibitors of apoptosis are being developed for numerous disor-

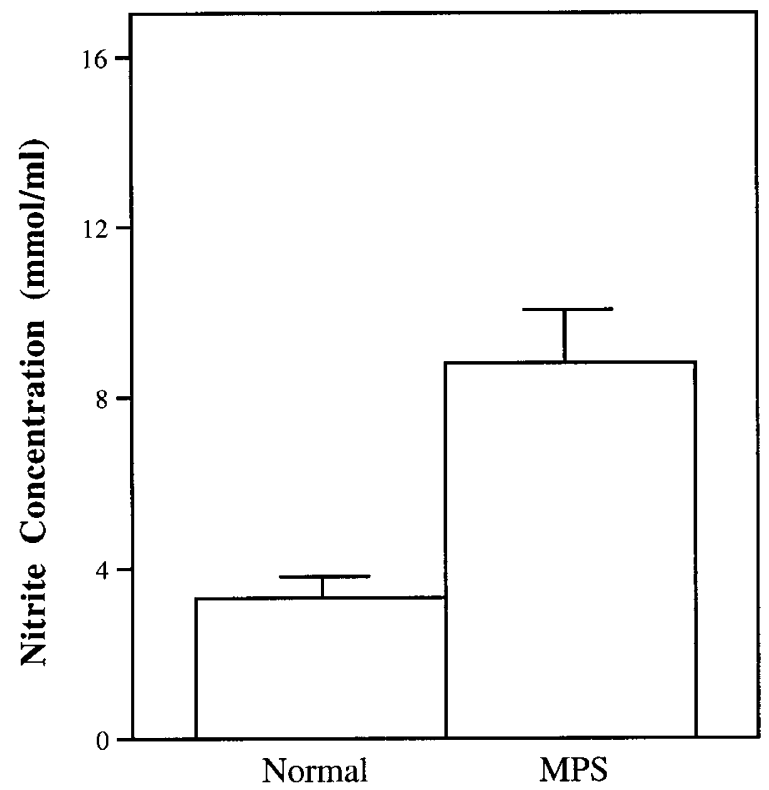

Figure 5.

NO formation in the media of MPS VI cat articular chondrocytes. Articular chondrocytes were obtained and grown from normal $(n=3)$ and MPS VI $(n$ $=6$ ) cats ranging in age from 6 months to 5 years, and the nitrite concentrations were determined in the culture media after 24 hours. The height of the columns indicates the mean values obtained from the duplicate analysis of individual animals, and the error bars show the standard error of the mean.

ders, including various forms of cancer and arthritis, and a further understanding of the mechanisms that lead to chondrocyte apoptosis in the MPS disorders would seem an important avenue of future research that might lead to new therapeutic approaches. In addition, traditional anti-inflammatory treatments may be useful in the management of these patients.

\section{Materials and Methods}

\section{Animals and Sample Isolation}

The MPS VI cat and rat models have been previously described (Jezyk et al, 1977; Yoshida et al, 1993). Affected and control animals were raised under $\mathrm{Na}-$ tional Institutes of Health and United States Department of Agriculture guidelines for the care and use of animals in research. The animals were housed with ad libitum food and water, 12-hour light cycles, at $21^{\circ} \mathrm{C}$, with 12 to 15 air changes per hour. Euthanasia was performed on cats using concentrated sodium pentobarbital in accordance with the American Veterinary Medical Association guidelines. Euthanasia of rats was performed using carbon dioxide inhalation. Breeding colonies were maintained under Institutional Animal Care and Use Committee (IACUC)-approved protocols and were genotyped by PCR-based methods. Humeri, femurs, and proximal tibia were collected from age-matched healthy and MPS animals and placed in either PBS for chondrocyte isolation or fixed in neutral buffered $10 \%$ formalin (Sigma, St. Louis, Missouri) for histological assessment (see below). The tissues were obtained from four animals in each age 


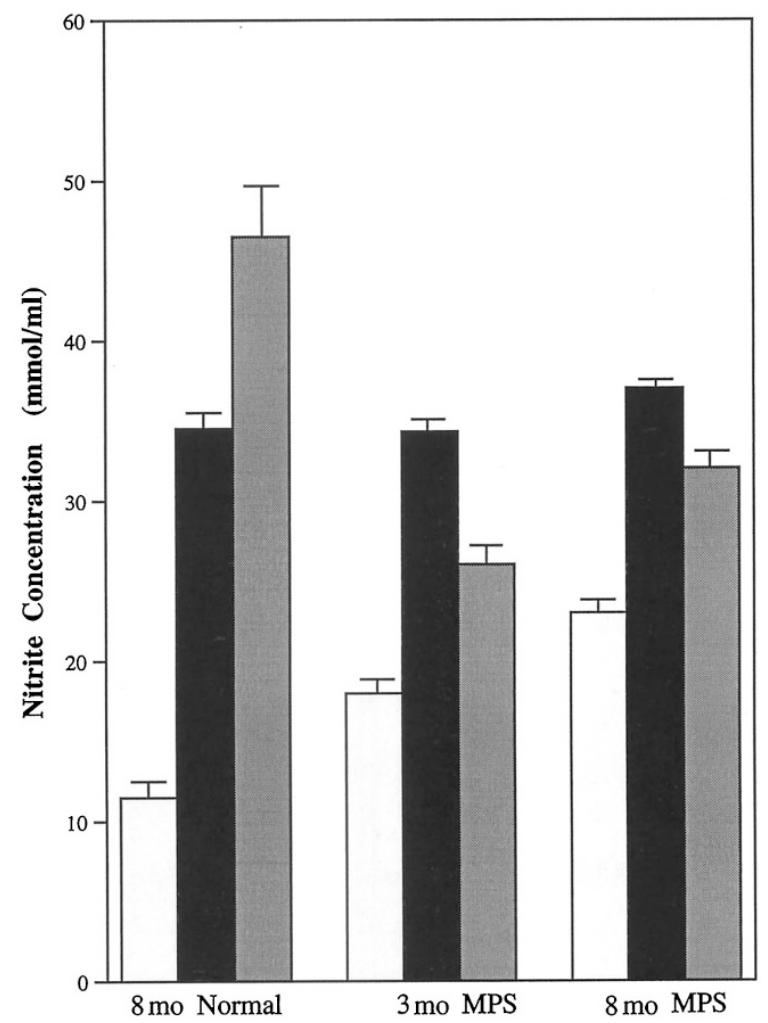

Figure 6.

Effects of lipopolysaccharide (LPS) and dermatan sulfate on NO formation in normal and MPS VI chondrocytes. Articular chondrocytes were obtained as described in "Materials and Methods," and grown in standard culture media (white boxes), media containing $100 \mu \mathrm{g} / \mathrm{ml}$ LPS (black boxes), or dermatan sulfate (gray boxes) for 24 hours. NO formation was then determined by quantifying nitrite production in the media using the Greiss reaction. For each age group, chondrocyte cultures from at least three different animals were analyzed in duplicate. The height of the columns indicates the mean values obtained and the error bars show the standard error of the mean.

group, ranging from 2 weeks to 12 months in the rats and from 3 to 60 months in the cats.

\section{Chondrocyte Isolation and Cross-Correction Studies}

To obtain chondrocytes the articular cartilage was sliced from the humeri, femoral heads, and condyles of the proximal tibia and washed in minimal essential medium (MEM; Gibco, Grand Island, New York). The cartilage was minced and digested in 15-ml conical tubes with $1 \%$ hyaluronidase for 1 hour at $37^{\circ} \mathrm{C}$, washed three times with PBS containing $1 \mathrm{~mm}$ EDTA, and sequentially incubated with $0.25 \%$ trypsin (Gibco) and $0.01 \mathrm{M}$ EDTA for 1 hour at $37^{\circ} \mathrm{C}$, followed by further digestion at $37^{\circ} \mathrm{C}$ for 8 hour with $0.2 \%$ collagenase (Sigma) prepared in MEM containing 10\% FCS. Cells were isolated by centrifugation $(450 \times g)$, washed twice, and plated in 96-well plates at a concentration of $7 \times 10^{4}$ cells in MEM containing $10 \%$ fetal bovine serum, 1\% glutamine, penicillin (150 units/ $\mathrm{ml})$, and streptomycin $(50 \mu \mathrm{g} / \mathrm{ml})$.

For the cross-correction studies, normal and MPS $\mathrm{VI}$ rat chondrocytes $\left(10^{7}\right.$ cells in $1 \mathrm{ml}$ of culture media) were plated into individual wells of Transwell coculture plates (Fisher Scientific, Morris Plains, New Jersey)

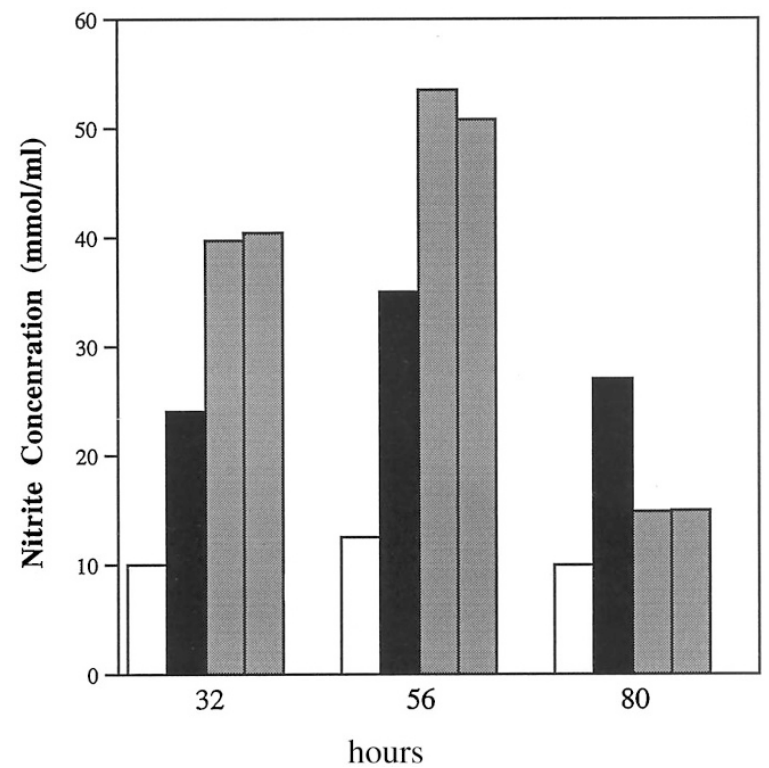

Figure 7.

NO levels in the media of cocultured normal and MPS VI chondrocytes. Articular chondrocytes were obtained from normal and MPS VI rats, and three sets of cocultures were established as described in "Materials and Methods": 1) both wells contained normal cells, white bars; 2) both wells contained MPS VI cells, black bars; and 3) each of the wells contained either MPS VI or normal cells, gray bars. The media was shared between the chondrocytes in each well, but the cells were never in direct contact. Each set of cocultures was established in duplicate. For sets 1 and 2 (controls), the height of the bars indicates the average NO values, whereas for set 3 (MPS VI and normal cocultures), the individual values for the duplicate experiments are shown. NO levels were determined at three time points after the cells were plated. Note that the NO levels in the media of MPS VI (black) cultures were approximately 2-fold greater than that found in normal (white) cultures, similar to the data shown in Figure 4. The initial NO levels in the media of the normal and MPS VI cocultures (gray) were approximately the sum of the two individual levels, but by 80 hours, these levels had been reduced to almost normal.

and grown for up to 80 hours. At various time points, $100 \mu$ of media was removed and the nitrite levels were determined as described below. The media that had been removed was replaced with fresh media to maintain a constant volume of $1 \mathrm{ml}$. Three cocultures were established: normal + normal; MPS VI + MPS VI, and normal + MPS VI.

\section{Histological Staining}

The formalin-fixed bones were decalcified in $8 \%$ formic acid (Sigma) for 5 days, embedded in paraffin, and sectioned $(5 \mu \mathrm{m})$ for histological analysis. Paraffinembedded tissues were deparaffinized and stained with hematoxylin and eosin (Sigma) and/or safranin-O (Sigma) and alcian blue (Sigma) for proteoglycan analysis (Hashimoto et al, 1999).

\section{Apoptosis Assessments}

Apoptosis in tissue sections and chondrocyte cultures was assessed by colorimetric and/or fluorometric DNA terminal transferase (TdT) nick-end translation methods (TUNEL assays). The colorimetric system measured nuclear DNA fragmentation by using TdT to add biotinylated nucleotides to the $3^{\prime}-\mathrm{OH}$ DNA ends and allowing 


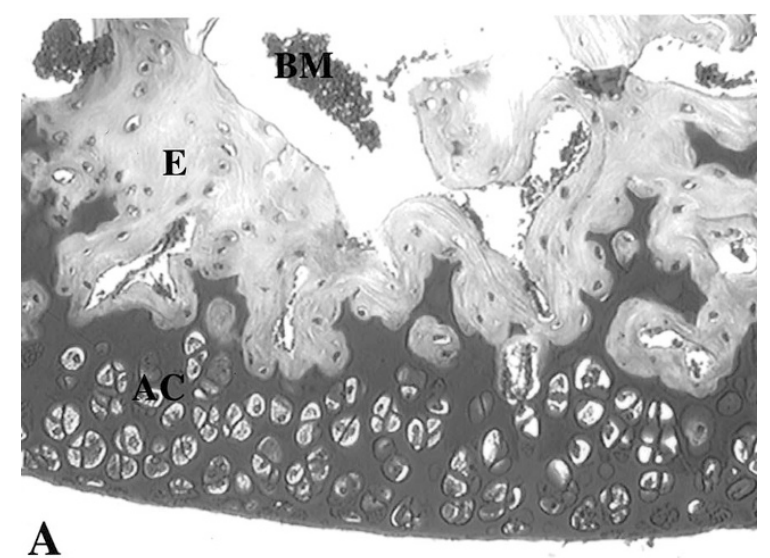

A

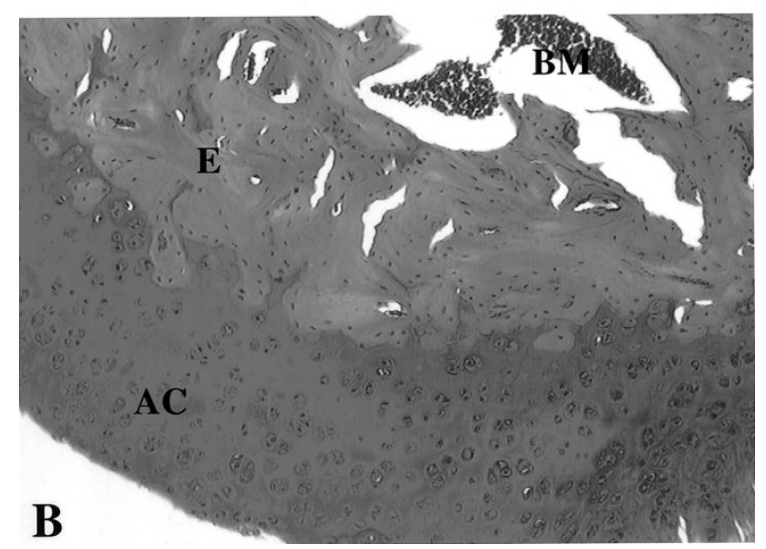

Figure 8.

Proteoglycan depletion in the articular cartilage of MPS VI rats. Longitudinal sections of 6 -month-old MPS VI (A) and normal (B) rat proximal tibia were stained with safranin-0 to visualize proteoglycans. BM, bone marrow; $\mathrm{E}$, epiphyses; AC, articular cartilage. Note that the epiphyses of the MPS VI rats stained less intensely than those of normal rats of the same age. Magnification, $\times 40$.

them to bind with horseradish peroxidase-labeled streptavidin (Promega, Madison, Wisconsin). Apoptotic nuclei were detected with peroxidase substrate (Sigma), hydrogen peroxide, and diaminobenzidine (DAB, Sigma) (Fuks et al, 1995). In the fluorescence-based TUNEL system (Promega), TdT added fluorescein-12-uridine triphosphate (UTP) to the $3^{\prime}-\mathrm{OH}$ ends of fragmented DNA. The fluorescently labeled DNA was visualized using a Nikon Eclipse E800 fluorescent microscope (Nikon, Tokyo, Japan). To determine the percentage of apoptotic cells in the tissue sections from each experimental group, a minimum of 200 cells/animal were counted and the percentage of TUNEL-positive cells was scored. For the cultured cells, 100 cells were counted and the percentage of TUNEL-positive cells scored.

Immunocytochemical staining using anti-PARP antibodies was used to confirm the results of the TUNEL assays. Tissue sections were deparaffinized in xylene, hydrated with $100 \%, 95 \%, 80 \%$, and $70 \%$ ethanol, and permeabilized in PBS containing $0.2 \%$ Triton-X-100 (Sigma). The sections were blocked for 2 hours at room temperature in PBS containing $0.1 \%$ Tween 20 (Sigma) and $5 \%$ FCS and then incubated overnight at $4^{\circ} \mathrm{C}$ in a

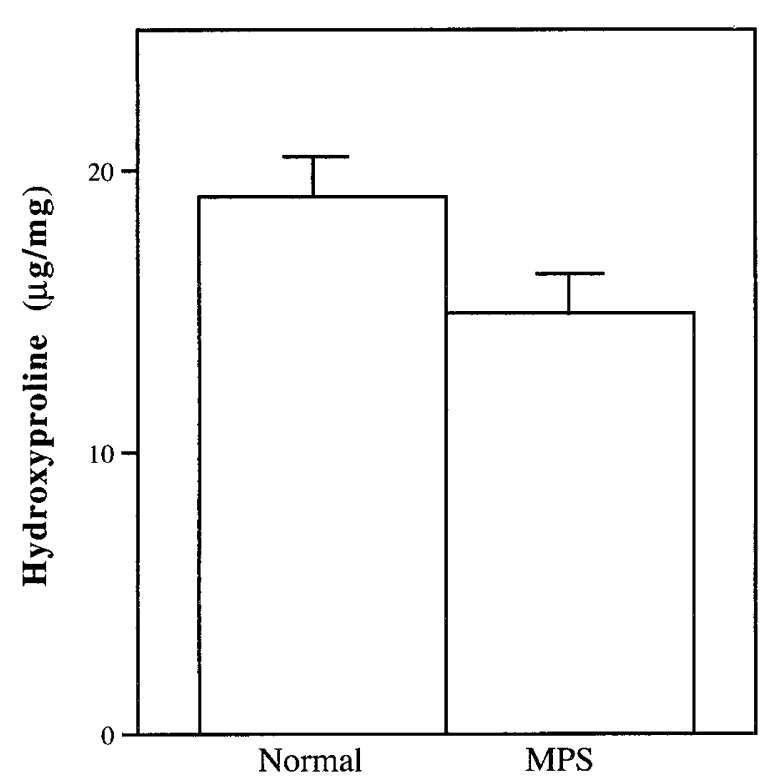

Figure 9.

Hydroxyproline content in normal and MPS VI articular cartilage. Articular cartilage from the proximal tibia of 6 -month-old healthy and MPS VI rats $(n=$ 3 each) was obtained and hydroxyproline was determined as described in "Materials and Methods" to estimate the total collagen content. Note that there was a significant reduction in the hydroxyproline content of MPS VI compared with normal cartilage ( $p$ value $<0.05$ )

humidified chamber with rabbit anti-PARP polyclonal antibodies specific for the $85 \mathrm{kd}$ fragment (p85) of human PARP (Promega). Sections were washed in $\times 1$ PBS, immersed in blocking buffer, and incubated for 2 hours at room temperature with a biotin-conjugated secondary antibody (rabbit Vectastain Elite ABC Kit; Vector Laboratories, Burlingame, California). Following incubation, the sections were washed and incubated with FITC-antihorseradish peroxidase (Accurate Chemical \& Scientific Corporation, Westbury, New York) for 1 hour at room temperature before being mounted on cover slips in Vectashield (Vector Laboratories).

\section{Nitrite and TNF- $\alpha$ Quantification}

Chondrocytes were isolated as described above, and $100 \mu \mathrm{g} / \mathrm{ml}$ of LPS (Sigma) or dermatan sulfate (Sigma) was added to the culture media. After 24 hours, the media was collected and the Griess reaction was used to detect nitrite accumulation (Green et al, 1982), a standard measure of NO formation. TNF- $\alpha$ quantification was performed on the rat media using a rat Quantikine ${ }^{\circledR}$ M immunoassay kit (R\&D Systems, Minneapolis, Minnesota).

\section{Hydroxyproline Quantification}

Articular cartilage from the proximal tibia of 6-monthold healthy and MPS VI rats ( $n=3$ each) was finely minced, washed twice with PBS and centrifuged. The pellet was dried, weighed (approximately $20 \mathrm{mg}$ ), and placed in a sealed hydrolysis vial at $105^{\circ} \mathrm{C}$ for 18 hours and processed for hydroxyproline content according to a previously described method (Firschein 


\section{Model For Degenerative Joint Disease In MPS Type VI}

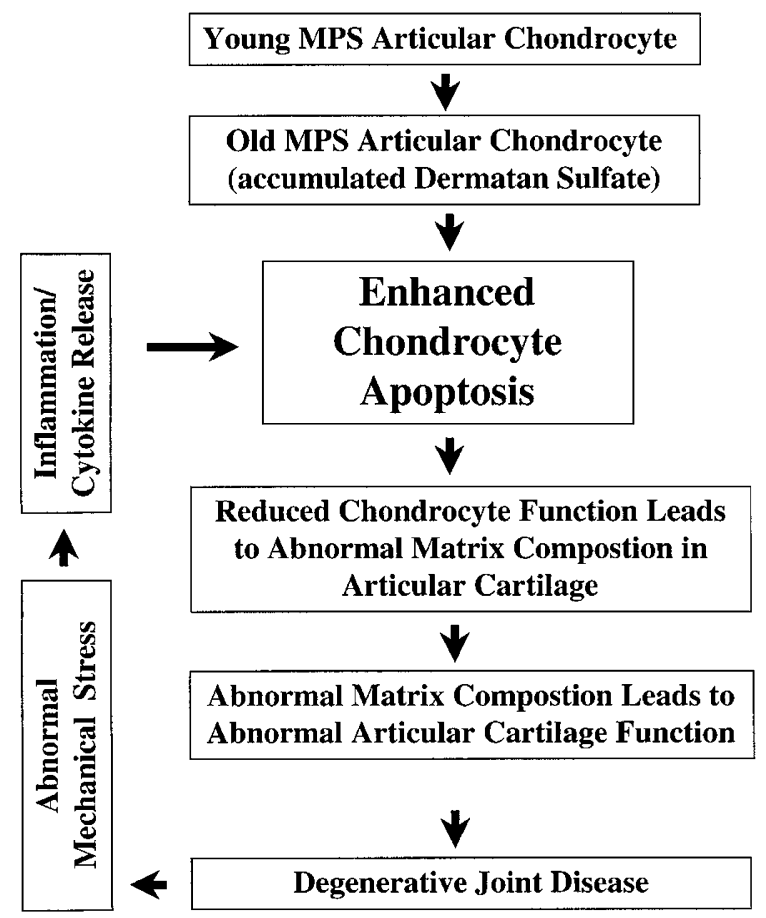

Figure 10.

Possible mechanism underlying degenerative joint disease in MPS VI. As MPS VI articular chondrocytes age, they accumulate and release excess amounts of dermatan sulfate-containing glycosaminoglycans. These cells eventually undergo apoptosis, caused by either the accumulating dermatan sulfate itself or cytokine-induced NO production resulting from mechanical stress on the MPS joints. The resulting MPS articular cartilage has an abnormal matrix composition that eventually causes functional deficits of the articular surfaces.

and Shill, 1966). Briefly, the hydrolyzed samples were neutralized with $\mathrm{NaOH}$ in $0.25 \mathrm{M}$ sodium citrate buffer containing $1 \mathrm{M}$ acetate, $\mathrm{pH} 6.0$, at $4^{\circ} \mathrm{C}$. A total of 100 $\mu \mathrm{l}$ of chloramine-T reagent containing $1.4 \%(\mathrm{w} / \mathrm{v})$ chloramine $\mathrm{T}, 20 \%(\mathrm{v} / \mathrm{v}) \mathrm{H}_{2} \mathrm{O}, 30 \%(\mathrm{v} / \mathrm{v})$ ethylene glycol monomethyl ether (EGME), and $50 \%$ (v/v) citrate buffer was added to $200 \mu \mathrm{l}$ of the sample, incubated for 20 minutes, and supplemented with 100 $\mu \mathrm{l}$ of $6.3 \mathrm{M}$ perchloric acid. After 5 minutes, $100 \mathrm{ml}$ of $20 \% \mathrm{w} / \mathrm{v} \mathrm{p}$-dimethylaminobenzaldehyde in EGME was added. The color was developed at $60^{\circ} \mathrm{C}$ for 20 minutes, cooled at room temperature, and read at 546 $\mathrm{nm}$. Hydroxyproline content from soluble collagen fragments was determined using pure hydroxyproline (Sigma) as a standard.

\section{Acknowledgements}

We wish to thank Nicole Vigna and Rongxiang Han for expert technical assistance, and Margaret Weil, Patricia O'Donnell, Jean Zweigle, and a cadre of veterinary students for compassionate animal care. We also wish to thank Zhenqiang Li for assistance with the hydroxyproline assays.

\section{References}

Abreu S, Hayden J, Berthold P, Shapiro IM, Decker S, Patterson D, and Haskins M (1995). Growth plate pathology in feline mucopolysaccharidosis VI. Calcif Tissue Int 57: 185-190.

Bielicki J, Crawley A, Davey R, Varnai J, and Hopwood J (1999). Advantages of using same species enzyme for replacement therapy in a feline model of mucopolysaccharidosis type VI. J Biol Chem 274:36335-363343.

Billinghurst RC, Dahlberg L, lonescu M, Reiner A, Bourne R, Rorabeck C, Mitchell P, Hambor J, Diekmann O, Tschesche H, Chen J, Van Wart H, and Poole AR (1997). Enhanced cleavage of type II collagen by collagenases in osteoarthritic articular cartilage. J Clin Invest 99:1534-1997.

Crawley AC, Niedzielski KH, Isaac EL, Davey RC, Byers S, and Hopwood JJ (1997). Enzyme replacement therapy from birth in a feline model of mucopolysaccharidosis type VI. $\mathrm{J}$ Clin Invest 99:651-652.

Daly TM, Vogler C, Levy B, Haskins ME, and Sands MS (1999). Neonatal gene transfer leads to widespread correction of pathology in a murine model of lysosomal storage disease. Proc Natl Acad Sci USA 96:2296-2300.

Firestein GS (1999). Signal transduction and transcription factors in rheumatic disease. Arthritis Rheum 42:609-621.

Firschein HE and Shill JP (1966). The determination of total hydroxyproline in urine and bone extracts. Anal Biochem 14:296-304.

Fuks Z, Alfieri A, Haimovitz-Friedman A, Seddon A, and Cordon-Cardo C (1995). Intravenous basic fibroblast growth factor protects the lung but not mediastinal organs against radiation-induced apoptosis in vivo. Cancer J Sci Am 1:6272.

Gerber HP, Vu TH, Ryan AM, Kowalski J, Werb Z, and Ferrara N (1999). VEGF couples hypertrophic cartilage remodeling, ossification and angiogenesis during endochondral ossification. Science 5:623-628.

Green LC, Wagner DA, Gologowski J, Skipper PL, Wishnok JS, and Tannenbaum SR (1982). Analysis of nitrate, nitrite, and [15N] nitrate in biological fluids. Anal Biochem 126:131138.

Hamerman D, Berman JW, Albers GW, Brown DL, and Silver D (1999). Emerging evidence for inflammation in conditions frequently affecting older adults: Report of a symposium. J Am Geriatr Soc 47:1016-1025.

Hashimoto S, Ochs RL, Komiya S, and Lotz M (1998). Linkage of chondrocyte apoptosis and cartilage degradation in human osteoarthritis. Arthritis Rheum 41:1632-1638.

Hashimoto S, Ochs RL, Rosen F, Quach J, McCabe G, Solan J, Seegmiller JE, Terkeltaub R, and Lotz M (1999). Chondrocyte-derived apoptotic bodies and calcification of articular cartilage. Proc Natl Acad Sci USA 95:3094-3099.

Jezyk PF, Haskins ME, Patterson DF, Mellman WJ, and Greenstein M (1977). Mucopolysaccharidosis in a cat with arylsulfatase B deficiency: A model of Maroteux-Lamy syndrome. Science 198:834-836.

Kakkis ED, McEntee MF, Schmidtchen A., Neufeld EF, Ward, Da, Gompf RE, Kania S, Bedolla C, Chien SL, and Shull RM (1996). Long-term and high-dose trials of enzyme replacement therapy in the canine model of mucopolysaccharidosis I. Biochem Mol Med 58:156-164. 
Lotz M (1999). The role of nitric oxide in articular cartilage damage. Rheum Dis Clin North Am 25:269-282.

Lotz M, Hashimoto S, and Kuhn K (1999). Mechanism of chondrocyte apoptosis. Osteoarthritis Cartilage 7:389-391.

Mayne R (1989). Cartilage collagens. What is their function, and are they involved in articular disease? Arthritis Rheum 32:241-246.

Neufeld EF and Muenzer J (2001). The Mucopolysaccharidoses. In: Scriver CR, editor. The Metabolic and Molecular Bases of Inherited Disease. New York: McGraw-Hill, 34213452.

Norrdin RW, Moffat KS, Thrall MA, and Gasper PW (1993). Characterization of osteopenia in feline mucopolysaccharidosis $\mathrm{VI}$ and evaluation of bone marrow transplantation therapy. Bone 14:361-367.

Ohashi T, Yokoo T, lizuka S, Kobayashi H, Sly W, and Eto Y (1999). Reduction of lysosomal storage in murine macrophages type VII by transplantation of normal and genetically modified macrophages. Blood 95:3631-3633.

Palmer RM, Hickery MS, Charles IG, Moncada S, and Bayliss MT (1993). Induction of nitric oxide synthase in human chondrocytes. Biochem Biophys Res Commun 193:398405.

Peters C, Shapiro EG, Anderson J, Henslee-Downey PJ, Klempeer MR, Cowan MJ, Saunders EF, deAlarcon PA, Twist C, Nachman JB, Hale GA, Harris RE, Rozans MK, Kurtzberg J, Grayson GH, Williams TE, Lenarsky C, Wagner JE, and Krivit W (1998). Hurler syndrome: II. Outcome of HLAgenotypically identical sibling and HLA-haploidentical related donor bone marrow transplantation in fifty-four children. The Storage Disease Collaborative Study Group. Blood 91:26012608.

Sakahira EM, Sakahira H, Yokoyama H, Okawa K, Iwamatsu A, and Nagata S (1998). A caspase-activated DNase that degrades DNA during apoptosis, and its inhibitor ICAD. Nature 391:43-50.
Santana P, Pena LA, Haimovitz-Friedman A, Seamus M, Green D, McLoughlin M, Cordon-Cardo C, Schuchman EH, Fuks Z, and Kolesnick R (1996). Acid sphingomyelinasedeficient human lymphoblasts and mice are defective in radiation induced apoptosis. Cell 86:189-199.

Simonaro CM, Haskins M, and Schuchman EH (1997). Bone marrow transplantation in newborn rats with mucopolysaccharidosis type VI. Transplantation 63:1386-1393.

Simonaro CM, Haskins ME, Abkowitz JL, Brooks DA, Hopwood JJ, Zhang J, and Schuchman EH (1999). Autologous transplantation of retrovirally transduced bone marrow or neonatal blood cells into cats can lead to long-term engraftment in the absence of myeloablation. Gene Ther 6:106-111.

Spiegel S, Foster D, and Kolesnick R (1996). Signal transduction through lipid second messengers. Curr Opin Cell Biol 8:159-167.

Stadler J, Stefanovic-Racic M, Billiar TR, Curran RD, Mclntyre LA, Georgescu HI, Simmons RL, and Evans CH (1991). Articular chondrocytes synthesize nitric oxide in response to cytokines and lipopolysaccharide. Immunology 1:39153920.

Turner C, Hopwood J, Bond C, and Brooks D (1999). Immune response to enzyme replacement therapy: 4-sulfatase epitope reactivity of plasma antibodies from MPS VI cats. Mol Genet Metab 67:194-205.

Yang C, Li SW, Helminen HJ, Khillan JS, Bao Y, and Prockop DJ (1997). Apoptosis of chondrocytes in transgenic mice lacking collagen II. Exp Cell Res 235:370-373.

Yoshida M, Noguchi J, Ikadai H, Takahashi M, and Nagase S (1993). Arylsulfatase B-deficient mucopolysaccharidosis in rats. J Clin Invest 91:1099-1104. 